\title{
ECOANATOMÍA DE LOS ELEMENTOS DE VASO DE LA MADERA DE CINCO ESPECIES DEL GÉNERO MIMOSA (LeGuMinOSAE-MimosoideAe)
}

\author{
Susana Adriana Montaño-Arias ${ }^{1,3}$, Sara lucía Camargo-Ricalde ${ }^{2}$ \\ y Carmen de la Paz Pérez-Olvera ${ }^{2}$ \\ ${ }^{1}$ Alumna del Doctorado en Ciencias Biológicas y de la Salud de la Universidad Autónoma Metropolitana, \\ México D.F., México \\ ${ }^{2}$ Departamento de Biología, División de Ciencias Biológicas y de la Salud, \\ Universidad Autónoma Metropolitana-Iztapalapa, México D.F., México \\ ${ }^{3}$ Autor para la correspondencia: arias_susan@hotmail.com
}

\begin{abstract}
Resumen: Mimosa es el género con mayor número de especies de Mimosoideae (Leguminosae) en México; se establecen desde el nivel del mar hasta 2,850 m de altitud. Se estimó la tolerancia de cinco especies de Mimosa al estrés hídrico, comparando algunas características anatómicas de los elementos de vaso del xilema secundario y se estimaron los índices de mesomorfía y de vulnerabilidad de la madera. Se elaboraron cortes transversales y preparaciones de material disociado de tres individuos por especie. Los índices de mesomorfía y de vulnerabilidad se estimaron considerando el diámetro de los vasos, el número de vasos/mm² y la longitud de los elementos de vaso. Las cinco especies presentan vasos numerosos (17 - 32), elementos de vaso cortos (150 - 221 $\mu \mathrm{m})$ y de diámetro pequeño (58 - $75 \mu \mathrm{m})$, excepto $M$. tenuiflora que presenta vasos de diámetro mediano (104 $\mu \mathrm{m})$. El índice de mesomorfía de la madera de las especies $>200$ corresponde a xilema mesofítico y el índice de vulnerabilidad $>1$ sugiere que las cinco especies son poco resistentes al estrés hídrico. El valor más alto del índice de vulnerabilidad corresponde a $M$. tenuiflora (6.26) y el más bajo a M. leucaenoides (1.81). Este tipo de estudios son importantes para la selección de especies vegetales con fines de restauración ambiental.
\end{abstract}

Palabras clave: ecoanatomía, estrés hídrico, madera, Mimosa.

\begin{abstract}
Mimosa is the genus with more species of Mimosoideae (Leguminosae) in Mexico; they establish from sea level to 2,850 m elevation. To estimate the tolerance to water stress of five Mimosa species, some wood anatomical characteristics of vessel elements were compared and the wood mesomorphy and vulnerability ratios calculated. Three plants per species were studied and transverse sections and macerations were prepared from the stem. The mesomorphy and vulnerability ratios were estimated considering vessel diameter, vessels $/ \mathrm{mm}^{2}$, and vessel element length. The five species showed numerous vessels (17 - 32) and short vessel elements $(150-221 \mu \mathrm{m})$ with small diameters (58 - $75 \mu \mathrm{m})$, except M. tenuiflora, with medium sized diameter (104 $\mu \mathrm{m})$. In the studied species, the mesomorphy ratio $>200$ matchs up to a mesomorphic wood and the vulnerability ratio $>1$ suggests a low resistant to water stress. The highest vulnerability ratio value corresponds to $M$. tenuiflora (6.26) and the lowest to M. leucaenoides (1.81). The results generated in this study are important to select plant species for ecological restoration purposes.
\end{abstract}

Key words: ecological anatomy, Mimosa, water stress, wood.

$\mathbf{L}$ as plantas están expuestas a factores ambientales cuya variación puede provocar cambios anatómicos para adaptar a la planta a las condiciones externas. En las plantas leñosas, las características de los elementos de vaso se modifican, dependiendo del grado de humedad del suelo y de factores como el clima, la altitud y la latitud (Wodzicki, 2001). Estudios de esta índole se han realizado comparando entre especies o entre individuos de una misma especie
(León-H., 2005). Aunque existen trabajos que registran la relación estructura de la madera con factores edáficos (Villagra y Roig-Juñent, 1997), son más numerosos los que reportan variaciones de la madera en función del clima (Baas, 1973; Carlquist, 1977; Dickison et al., 1978; Oever et al., 1981; Baas et al., 1983; Sidiyasa y Bass, 1998; Bernal-Salazar y Terrazas, 2000; Arias y Terrazas, 2001; Aguilar-Rodríguez et al., 2006; Moya y Tomazello, 2008; de Lima et 
al., 2009; Chávez-Romero et al., 2010). Con respecto a los elementos de vaso, se han propuesto índices para evaluar la vulnerabilidad a la sequía; por ejemplo, Carlquist (1977) estimó estos índices en plantas de sitios con diferente precipitación (p. ej. desierto, pantano), estableciendo que una planta con un índice de vulnerabilidad (IV) $>1$ es vulnerable al estrés hídrico y si presenta un índice de mesomorfía (IM) > 200 tendrá un xilema mesofítico y por debajo de los 75 es considerado xerófito. Este tipo de estudios permite conocer la resistencia de las especies a una baja precipitación, principalmente en plantas que habitan en zonas secas.

En las zonas donde el agua es uno de los factores limitantes, las leguminosas se encuentran entre los elementos dominantes (De la Barrera y Andrade, 2005) y están bien representadas en México (Sosa y Dávila, 1994). Por su parte, Mimosa es el género más diverso de Mimosoideae en el país, con aproximadamente 110 especies, de las cuales cerca del 60\% son endémicas (Grether et al., 1996). Asimismo, varias especies de Mimosa se consideran plantas multipropósito (Camargo-Ricalde et al., 2001) e incluso son plantas potencialmente útiles para restaurar ambientes semiáridos (Dhillion et al., 2004; Martínez-Pérez et al., 2006; Pavón et al., 2011).

En México, se han realizado trabajos descriptivos sobre la anatomía de la madera de varias especies de Mimosa (Barajas-Morales y León-Gómez, 1989; Chehaibar y Grether, 1990; Camargo-Ricalde, 2000). Recientemente, MontañoArias (2010) determinó los caracteres anatómicos con valor taxonómico que permiten separar o agrupar a las especies de Mimosa sección Batocaulon. A pesar de que existen estudios ecoanatómicos y ecofisiológicos que permiten conocer algunas correlaciones entre los factores ambientales y algunos caracteres anatómicos de la madera en Leguminosae, en Mimosa no se registran, por lo que todavía se carece de información para estimar la tolerancia de las especies de este género al estrés hídrico. A nivel de la familia Leguminosae, destaca el estudio de Carlquist (1977), quien estimó el IV para nueve especies de Acacia y otras cuatro especies de leguminosas (Bossiaea laidlawiana Tovey \& Morris, Hovea elliptica (Sm.) DC., Templetonia retusa (Vent.) R.Br. y Pultenaea dasyphylla Turcz.), caracterizándolas como especies resistentes al estrés hídrico y con xilema xerófito. Moglia y Giménez (1998) interpretaron las estrategias adaptativas del xilema de 19 especies correspondientes a diferentes familias (p. ej. Boraginaceae, Leguminosae, Simarubaceae y Ulmaceae). Ellos determinaron los IV de dos mimosoideas Acacia y Prosopis, así como de Erythrina crista-galli L., Chorisia insignis HBK y Ch. speciosa A. St.-Hill., registrando para todas las especies vulnerabilidad a la sequía. Por su parte, Giménez (2004) describe la madera de dos leguminosas, Geoffroea decorticans Burkart y G. striata Morong, señalando que, como en los casos anteriores, ambas son vulnerables al estrés hídrico por presentar un IV de 3.8 y de 2.1, respectivamente. Recientemente, Quintanar-Isaías et al. (2009) determinaron que Gliricidia sepium (Jacq.)
Steud presenta un IV de 95 y un IM superior a 200 (= xilema mesofítico), esto refleja su baja resistencia a la sequía, lo que corresponde con los climas semihúmedos en los que esta especie se desarrolla.

Otro carácter relacionado con la tolerancia al estrés hídrico o sequía, es el número de vasos por milímetro cuadrado $\left(\operatorname{vasos} / \mathrm{mm}^{2}\right.$ ), un alto número de vasos $/ \mathrm{mm}^{2}$ (p. ej. 251) es frecuentemente registrado en plantas de ambientes secos (Carlquist y Hoekman, 1985, Wilkings y Papassotiriou, 1989) o fríos (Miller, 1975). Por su parte, las especies de regiones húmedas se caracterizan por presentar un xilema con elementos de vaso largos, diámetros medianos a grandes y poco numerosos para conducir grandes volúmenes de agua en poco tiempo. Todo lo anterior demuestra que, dependiendo del hábitat de las especies, serán las estrategias que desarrollen las plantas para optimizar y asegurar la conducción de agua (Moglia y Giménez, 1998).

No obstante, existen otros caracteres que dan información a cerca de la tolerancia de las especies a la sequía, como es el caso de los vasos agrupados que confieren una mayor seguridad en la conducción de agua (Moglia y Giménez, 1998). La presencia de placas de perforación simple ofrece una menor resistencia de los vasos a la cavitación (Ellerby y Ennos, 1998). Asimismo, Jansen et al. (2003) y Choat et al. (2004) sugieren que las especies que habitan en ambientes secos presentan punteaduras intervasculares ornamentadas, esto para prevenir la ruptura de la membrana de la punteadura del elemento de vaso cuando hay pérdida de la presión de agua (Zweypfenning, 1978; Carlquist, 1982, Choat et al., 2004).

El objetivo de este estudio fue comparar algunas características morfológicas de los elementos de vaso y estimar el IV e IM de cinco especies de Mimosa, con la finalidad de evaluar su tolerancia a la sequía. De acuerdo con los escenarios del Panel Intergubernamental de Cambio Climático (IPCC) (Christensen et al., 2007), la temperatura aumentará de 0.3 a $5{ }^{\circ} \mathrm{C}$ en los próximos cien años, lo que alterará los regímenes de precipitación y, en consecuencia, la humedad del suelo, lo que repercutirá en la composición y diversidad de los ecosistemas secos de México.

\section{Materiales y métodos}

Se eligieron cinco especies arbóreas del género Mimosa que crecen en diversas condiciones ambientales en México: $M$. bahamensis Benth., M. hexandra M.Micheli, M. leucaenoides Benth., M. tejupilcana R.Grether \& A.Martinez-Bernal y $M$. tenuiflora (Willd.) Poir. Las cinco especies son caducifolias.

Para tener representada la variabilidad de cada especie, se recolectaron tres árboles por especie. Los ejemplares de referencia y las tablillas correspondientes están depositados en el Herbario Metropolitano Dr. Ramón Riba y Nava Esparza (UAMIZ), de la Universidad Autónoma Metropolitana, Unidad Iztapalapa (Cuadro 1). 
Para cada sitio de colecta, se anotaron las coordenadas geográficas y datos de los individuos (altura y diámetro del tronco, Cuadro 1). Se tomaron muestras del tronco a $80 \mathrm{~cm}$ del suelo. Con un micrótomo de deslizamiento (American Optical, modelo 860), se cortaron secciones transversales con grosor de $20 \mu \mathrm{m}$; se tiñeron con safranina-verde rápido y se montaron con resina sintética (Johansen, 1940). Se colocaron astillas de la sección radial en un tubo de ensayo con una mezcla, a partes iguales, de ácido acético, láctico y nítrico, y glicerina, dejándolos reposar por 1-2 semanas. Una vez disociado el material, éste se lavó y se hicieron preparaciones temporales para cuantificar la longitud de los elementos de vaso. Para la descripción anatómica de la madera, se siguió la nomenclatura propuesta por el IAWA Committee (1989). Se realizaron 25 mediciones para cada característica de los elementos de vaso (número de vasos/ $\mathrm{mm}^{2}$; longitud, diámetro y grosor de la pared) por individuo por especie. El número de vasos $/ \mathrm{mm}^{2}$ se contabilizó tomando en cuenta vasos solitarios y agrupados. En los vasos que se encuentran agrupados, cada vaso se contabilizó como vaso solitario. El grosor de la pared del elemento de vaso se midió en corte transversal, mientras que el diámetro de la punteadura intervascular que abarca todo el cuerpo de la punteadura; así como el diámetro de la cámara (lumen) de la punteadura en el material disociado. Las mediciones se realizaron utilizando un microscopio óptico (Zeiss modelo Axiostar-Plus).

El diámetro de los vasos se consideró grande cuanto éste midió $(>200 \mu \mathrm{m})$, mediano $(100-200 \mu \mathrm{m})$ y pequeño $(<$ $25 \mu \mathrm{m} \mathrm{a}<100 \mu \mathrm{m}$, IAWA Committee, 1989). Además, el diámetro de los vasos (dv) y el número de vasos $/ \mathrm{mm}^{2}$ (nv) se emplearon para calcular el Índice de Vulnerabilidad, IV = $\mathrm{dv} / \mathrm{nv}$; donde un valor inferior a 1 se interpreta como resistente a la sequía (Carlquist, 1977). La longitud del elemento de vaso (lv) y el IV se emplearon para calcular el Índice de Mesomorfía; el IM = IV ·lv, un IM mayor de 200 indica que el xilema es mesofítico y un IM menor o igual a 75 indica un xilema xerófito (Carlquist, 1977).

Los datos obtenidos se examinaron por medio de un análisis de varianza (ANOVA), seguido por la prueba de TukeyKramer $(P \leq 0.05)$, con el objeto de evaluar la existencia de diferencias estadísticamente significativas entre los caracteres evaluados intra- e inter-específicamente (Sokal y Rohlf, 1995). Cabe mencionar que a nivel intraespecífico, no se encontraron diferencias significativas.

Los valores de IV e IM no se ajustaron a la normal, por lo que se realizó un análisis de varianza de Kruskal-Wallis, seguido por la prueba de Mann-Whitney $(P \leq 0.05)$ con el fin de evaluar la existencia de diferencias estadísticamente significativas entre especies (Sokal y Rohlf, 1995).

Además, se analizó la asociación entre cuatro caracteres cuantitativos de los elementos de vaso y la altitud mediante un análisis de correlación lineal simple de Pearson (Cuadro 3). Los análisis estadísticos se llevaron a cabo mediante el paquete estadístico NCSS (Hintze, 2001).

\section{Resultados}

Las cinco especies de Mimosa presentan porosidad difusa con anillos de crecimiento (Figura 1). En Mimosa baha-

Cuadro 1. Estado, tipo de vegetación, clima, localización, altitud de los sitios de recolección de las especies de Mimosa (Leguminosae-Mimosoideae) estudiadas y número de registro de herbario y xiloteca (UAMIZ); así como la altura y el diámetro a la altura del pecho (DAP) de los individuos seleccionados.

\begin{tabular}{|c|c|c|c|c|c|c|c|}
\hline Especie & Estado & Tipo de vegetación & $\begin{array}{c}\text { Clima } \\
\text { (García, 1973) }\end{array}$ & $\begin{array}{l}\text { Coordenadas/ } \\
\text { Altitud } \\
\text { (m s.n.m.) }\end{array}$ & $\begin{array}{c}\text { Herbario } \\
\text { (UAMIZ) } \\
\text { No. Registro }\end{array}$ & $\begin{array}{c}\text { Xiloteca } \\
\text { (UAMIZ) } \\
\text { No. Registro }\end{array}$ & $\begin{array}{c}\text { Altura }(\mathbf{m}) / \\
\operatorname{DAP}(\mathbf{c m})\end{array}$ \\
\hline $\begin{array}{c}\text { M. } \\
\text { bahamensis }\end{array}$ & Yucatán & $\begin{array}{l}\text { Bosque tropical } \\
\text { caducifolio }\end{array}$ & $\begin{array}{l}\text { Seco con lluvias } \\
\text { poco abundantes } \\
\text { en todas las } \\
\text { estaciones }\left(\mathrm{BS}_{0}\right)\end{array}$ & $\begin{array}{c}21^{\circ} 11.284^{\prime} \mathrm{N} \\
89^{\circ} 48.125^{\prime} \mathrm{O} / 110\end{array}$ & $\begin{array}{l}65773 \\
65774 \\
65776\end{array}$ & $\begin{array}{l}\text { UAMIZ- } \\
\text { M257,258, } \\
259\end{array}$ & $\begin{array}{l}3,4,3 / \\
7,6,7\end{array}$ \\
\hline M. hexandra & Oaxaca & Matorral xerófilo & $\begin{array}{c}\text { Cálido subhúmedo } \\
\text { con Iluvias en } \\
\text { verano }\left(A w_{1}\right)\end{array}$ & $\begin{array}{c}16^{\circ} 34.285^{\prime} \mathrm{N} ; \\
94^{\circ} 56.852^{\prime} \mathrm{O} / 73\end{array}$ & $\begin{array}{l}64622 \\
64616 \\
64618\end{array}$ & $\begin{array}{l}\text { UAMIZ- } \\
\text { M260,261, } \\
262\end{array}$ & $\begin{array}{c}3,3,2.5 / \\
5,7,8\end{array}$ \\
\hline $\begin{array}{c}\text { M. } \\
\text { leucaenoides }\end{array}$ & Tamaulipas & $\begin{array}{c}\text { Zona de transición } \\
\text { entre bosque tropical } \\
\text { perennifolio y } \\
\text { matorral xerófilo }\end{array}$ & $\begin{array}{c}\text { Templado } \\
\text { subhúmedo con } \\
\text { Iluvias en verano } \\
\left(\mathrm{Cw}_{1}\right)\end{array}$ & $\begin{array}{c}23^{\circ} 18.956^{\prime} \mathrm{N} ; \\
99^{\circ} 40.157^{\prime} \mathrm{O} / \\
1730\end{array}$ & $\begin{array}{l}64145 \\
64143 \\
64148\end{array}$ & $\begin{array}{l}\text { UAMIZ- } \\
\text { M254,255, } \\
256\end{array}$ & $\begin{array}{l}3.2,3,3 / \\
6.5,5,6\end{array}$ \\
\hline $\begin{array}{c}M . \\
\text { tejupilcana }\end{array}$ & $\begin{array}{l}\text { Estado de } \\
\text { México }\end{array}$ & Bosque de Quercus & $\begin{array}{c}\text { Cálido subhúmedo } \\
\text { con Iluvias en } \\
\text { verano }\left(A w_{1}\right)\end{array}$ & $\begin{array}{c}18^{\circ} 52.905^{\prime} \mathrm{N} ; \\
100^{\circ} 18.109^{\prime} \mathrm{O} / \\
1650\end{array}$ & $\begin{array}{l}64400 \\
64408 \\
64401\end{array}$ & $\begin{array}{l}\text { UAMIZ- } \\
\text { M266,267, } \\
268\end{array}$ & $\begin{array}{l}2,2,2 / \\
2,2,2\end{array}$ \\
\hline M. tenuiflora & Oaxaca & $\begin{array}{l}\text { Bosque tropical } \\
\text { caducifolio }\end{array}$ & $\begin{array}{c}\text { Cálido subhúmedo } \\
\text { con lluvias en } \\
\text { verano }\left(A w_{1}\right)\end{array}$ & $\begin{array}{c}16^{\circ} 31.118^{\prime} \mathrm{N} ; \\
94^{\circ} 30.724^{\prime} \mathrm{O} / \\
130\end{array}$ & $\begin{array}{l}64621 \\
64619 \\
64622\end{array}$ & $\begin{array}{l}\text { UAMIZ- } \\
\text { M263,264, } \\
265\end{array}$ & $\begin{array}{l}3,5,6 / \\
5,7,8\end{array}$ \\
\hline
\end{tabular}



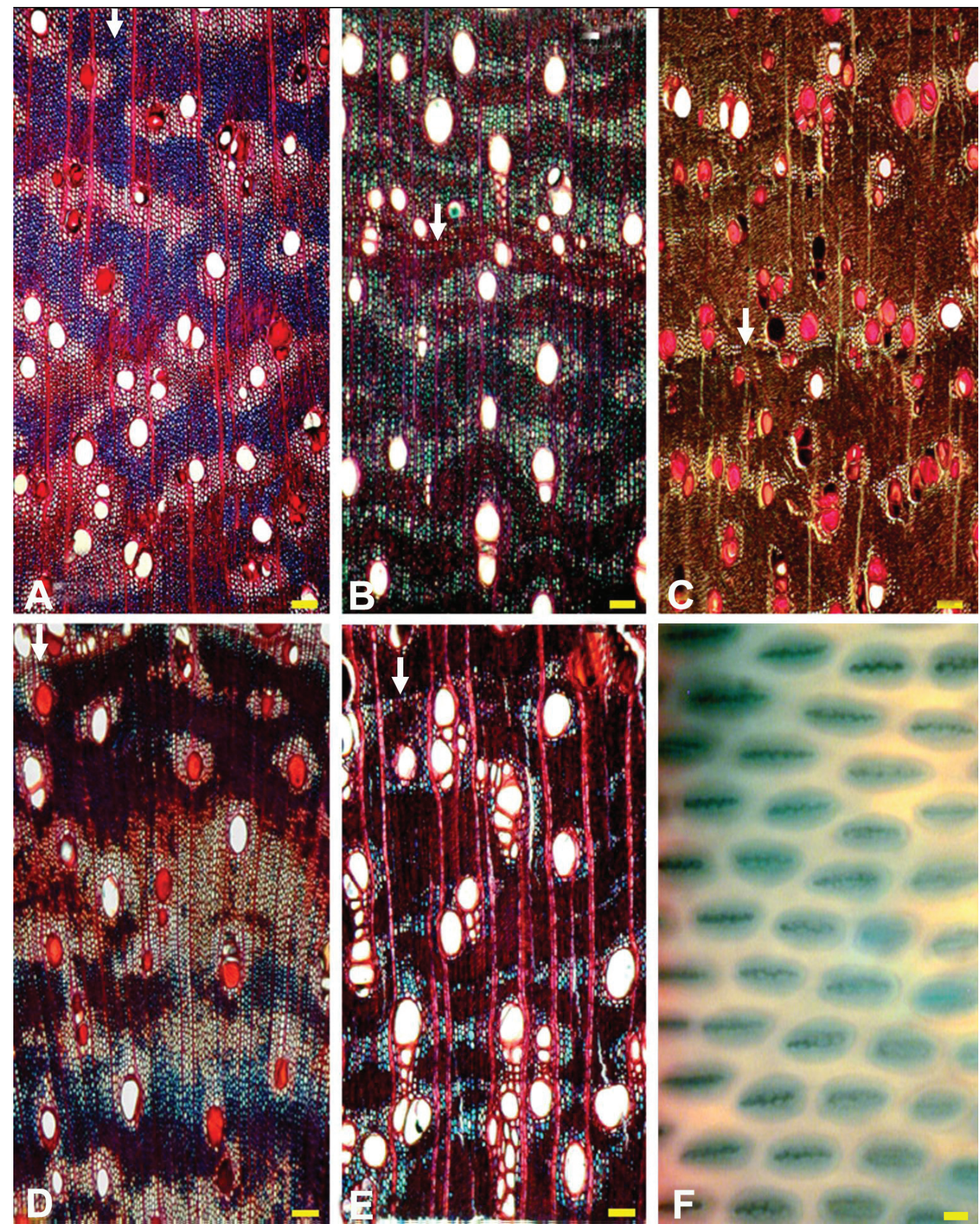

Figura 1. Cortes transversales, anillos de crecimiento, porosidad y vasos agrupados. A. Mimosa bahamensis, B. M. hexandra, C. M. leucaenoides, D. M. tejupilcana, E. M. tenuiflora, F. Punteaduras intervasculares alternas y ornamentadas carácter común en las cinco especies estudiadas. Las flechas indican el inicio de un anillo de crecimiento. A-E barra $=100 \mu \mathrm{m}, \mathrm{F}$ barra $=5 \mu \mathrm{m}$. 
mensis, M. leucaenoides, $M$. tejupilcana y M. tenuiflora, los anillos de crecimiento están delimitados por una o hasta 12 hileras de fibras con paredes engrosadas. Mientras que en $M$. hexandra, están delimitados por cinco hileras de fibras con paredes gruesas y una banda de parénquima.

La madera de las especies presenta vasos numerosos (> 10 vasos $/ \mathrm{mm}^{2}$ ), Mimosa leucaenoides presenta el mayor número de vasos $/ \mathrm{mm}^{2}$, lo que la distingue del resto de las especies (Cuadro 2). Además, las cinco especies presentan vasos agrupados. No obstante, $M$. tejupilcana sólo presentó agrupaciones de dos vasos; M. bahamensis y $M$. hexandra dos y tres vasos; mientras que en $M$. leucaenoides y M. tenuiflora se observaron de dos a cinco vasos agrupados (Figura 1).

Descripción de los elementos de vaso. Respecto al grosor de la pared y al diámetro tangencial del vaso, Mimosa tenuiflora se distingue por presentar los vasos de mayor diámetro y de pared más gruesa, existiendo diferencias significativas entre M. bahamensis, M. hexandra, M. leucaenoides y M. tejupilcana (Cuadro 2). Al comparar entre M. hexandra y M. tejupilcana no existen diferencias significativas con relación al grosor de la pared y al número de vasos $/ \mathrm{mm}^{2}$. Sin embargo,

Cuadro 2. Resultados del análisis de la estadística descriptiva y el análisis de varianza (ANOVA) y Kruskal-Wallis, para los índices de vulnerabilidad y mesomorfía de las cinco especies de Mimosa (Leguminosae-Mimosoideae) estudiadas. Los valores seguidos por la misma letra en forma vertical no difieren significativamente $(P \geq 0.05)$; mientras que los valores seguidos por letras diferentes, muestran diferencias significativas $(P<0.05) . \varnothing=$ Diámetro.

\begin{tabular}{|c|c|c|c|c|c|c|}
\hline \multirow[t]{2}{*}{ Variables } & \multirow[t]{2}{*}{ Especies } & \multirow{2}{*}{$\begin{array}{c}\text { Media } \pm \text { desviación } \\
\text { estándar }\end{array}$} & \multirow{2}{*}{$\begin{array}{l}\text { Valor } \\
\text { mínimo }\end{array}$} & \multirow{2}{*}{$\begin{array}{l}\text { Valor } \\
\text { máximo }\end{array}$} & \multicolumn{2}{|c|}{ ANOVA } \\
\hline & & & & & $F$ & $P$ \\
\hline \multirow[t]{5}{*}{ Vasos/ mm² } & M. bahamensis & $23 \pm 1.67^{b}$ & 11 & 30 & \multirow[t]{5}{*}{23.35} & \multirow[t]{5}{*}{$0.000047^{*}$} \\
\hline & M. hexandra & $20 \pm 1.64^{\mathrm{b}}$ & 11 & 27 & & \\
\hline & M. leucaenoides & $32 \pm 1.24^{\mathrm{a}}$ & 22 & 41 & & \\
\hline & M. tejupilcana & $17 \pm 0.82^{b}$ & 7 & 27 & & \\
\hline & M. tenuiflora & $17 \pm 4.21^{b}$ & 10 & 30 & & \\
\hline \multirow{5}{*}{$\begin{array}{l}\varnothing \text { tangencial } \\
\text { del vaso }(\mu \mathrm{m})\end{array}$} & M. bahamensis & $75 \pm 4.0^{\mathrm{b}}$ & 37 & 117 & \multirow[t]{5}{*}{29.14} & \multirow[t]{5}{*}{$0.000017^{*}$} \\
\hline & M. hexandra & $72 \pm 3.0 c^{b}$ & 45 & 110 & & \\
\hline & M. leucaenoides & $58 \pm 4^{c}$ & 37 & 90 & & \\
\hline & M. tejupilcana & $68 \pm 5.0^{\mathrm{cb}}$ & 35 & 105 & & \\
\hline & M. tenuiflora & $104 \pm 9.01^{\mathrm{a}}$ & 62 & 157 & & \\
\hline \multirow{5}{*}{$\begin{array}{l}\text { Grosor de la } \\
\text { pared }(\mu \mathrm{m})\end{array}$} & M. bahamensis & $7 \pm 0.38^{b}$ & 5 & 10 & \multirow[t]{5}{*}{38.85} & \multirow[t]{5}{*}{$0.000005^{*}$} \\
\hline & M. hexandra & $7 \pm 0.06^{b}$ & 5 & 12 & & \\
\hline & M. leucaenoides & $5 \pm 0.15^{c}$ & 5 & 7 & & \\
\hline & M. tejupilcana & $5 \pm 0.35^{c}$ & 5 & 7 & & \\
\hline & M. tenuiflora & $8 \pm 0.76^{a}$ & 5 & 12 & & \\
\hline \multirow{5}{*}{$\begin{array}{l}\text { Longitud de } \\
\text { los elementos } \\
\text { vaso }(\mu \mathrm{m})\end{array}$} & M. bahamensis & $185 \pm 11.0^{\mathrm{ab}}$ & 70 & 320 & \multirow[t]{5}{*}{7.84} & \multirow[t]{5}{*}{$0.003952 *$} \\
\hline & M. hexandra & $150 \pm 34.0^{b}$ & 65 & 275 & & \\
\hline & M. leucaenoides & $152 \pm 8^{\mathrm{b}}$ & 60 & 200 & & \\
\hline & M. tejupilcana & $177 \pm 14 .^{\mathrm{ab}} 0$ & 70 & 250 & & \\
\hline & M. tenuiflora & $221 \pm 5.83^{a}$ & 100 & 350 & & \\
\hline \multirow{5}{*}{$\begin{array}{c}\varnothing \text { de la punteadura } \\
\text { intervascular } \\
(\mu \mathrm{m})\end{array}$} & M. bahamensis & $5 \pm 0.17^{b c}$ & 5 & 7 & \multirow[t]{5}{*}{10.94} & \multirow[t]{5}{*}{$0.000047^{*}$} \\
\hline & M. hexandra & $5 \pm 0.55^{c}$ & 2 & 7 & & \\
\hline & M. leucaenoides & $6 \pm 0.25^{b c}$ & 2 & 10 & & \\
\hline & M. tejupilcana & $7 \pm 0.17^{a}$ & 5 & 12 & & \\
\hline & M. tenuiflora & $6 \pm 0.32^{b}$ & 5 & 12 & & \\
\hline \multirow{5}{*}{$\begin{array}{c}\varnothing \text { de la cámara } \\
\text { de la punteadura } \\
\text { intervascular }(\mu \mathrm{m})\end{array}$} & M. bahamensis & $3 \pm 0.20^{d}$ & 2 & 5 & \multirow[t]{5}{*}{13.07} & \multirow[t]{5}{*}{$0.000015^{*}$} \\
\hline & M. hexandra & $3 \pm 0.26^{\text {bcd }}$ & 2 & 7 & & \\
\hline & M. leucaenoides & $3 \pm 0.06^{\mathrm{bc}}$ & 2 & 7 & & \\
\hline & M. tejupilcana & $4 \pm 0.21^{a}$ & 2 & 10 & & \\
\hline & M. tenuiflora & $3 \pm 0.20^{\mathrm{bcd}}$ & 2 & 5 & & \\
\hline \multirow{5}{*}{$\begin{array}{c}\text { Índice de } \\
\text { vulnerabilidad (IV) }\end{array}$} & M. bahamensis & $3.36 \pm 0.15^{b}$ & 3.19 & 3.48 & $H$ & $0.009552 *$ \\
\hline & M. hexandra & $3.56 \pm 0.13^{b}$ & 3.48 & 3.71 & 13.3822 & \\
\hline & M. leucaenoides & $1.81 \pm 0.05^{c}$ & 1.75 & 1.86 & & \\
\hline & M. tejupilcana & $4.09 \pm 0.15^{b}$ & 3.93 & 4.24 & & \\
\hline & M. tenuiflora & $6.26 \pm 1.66^{\mathrm{a}}$ & 4.35 & 7.38 & & \\
\hline Índice de & M. bahamensis & $621.92 \pm 36.06^{\mathrm{ab}}$ & 586.46 & 658.56 & $H$ & $0.011114^{*}$ \\
\hline mesomorfía (IM) & M. hexandra & $537.22 \pm 128.83^{b}$ & 388.54 & 615.79 & 13.03333 & \\
\hline & M. leucaenoides & $276.77 \pm 22.39^{c}$ & 251.67 & 294.67 & & \\
\hline & M. tejupilcana & $727.76 \pm 83.69^{b}$ & 668.16 & 823.44 & & \\
\hline & M. tenuiflora & $1380.52 \pm 339.61^{\mathrm{a}}$ & 991.88 & 1620.19 & & \\
\hline
\end{tabular}


M. tejupilcana mostró diferencias significativas únicamente con relación al diámetro tangencial del vaso comparada con $M$. tenuiflora. Es relevante mencionar que M. leucaenoides presentó el menor diámetro de vaso (Figura1).

Longitud de elementos de vaso, placas de perforación y punteaduras intervasculares. Las cinco especies presentaron elementos de vaso cortos (150 - $221 \mu \mathrm{m})$. En este carácter no existen diferencias significativas entre Mimosa bahamensis, M. tejupilcana y M. tenuiflora, pero sí al comparar entre estas tres especies con $M$. hexandra; por su parte, $M$. leucaenoides sólo mostró diferencia significativa al compararse con M. tenuiflora (Cuadro 2).

Las especies presentaron elementos de vaso con placa de perforación simple, punteaduras intervasculares areoladas, alternas y ornamentadas. Mimosa tejupilcana presenta las de mayor diámetro; mientras que $M$. hexandra presenta las punteaduras de menor diámetro. Entre $M$. bahamensis, $M$. hexandra y M. leucaenoides no existen diferencias significativas en cuanto al diámetro de la punteadura intervascular. El diámetro menor de la cámara de las punteaduras intervasculares lo presentó M. bahamensis (Cuadro 2, Figura 1).

Índices de Vulnerabilidad (IV) y Mesomorfía (IM). Los IV obtenidos varían entre 1.81 y 6.26 (Cuadro 2). El valor más bajo de IV lo presentó Mimosa leucaenoides, lo que indica una menor vulnerabilidad a la sequía, seguido de $M$. bahamensis $<M$. hexandra $<M$. tejupilcana $<M$. tenuiflora, esta última presentó el mayor IV, lo que indica que es la más vulnerable a la sequía. Sin embargo, entre $M$. bahamensis, $M$. hexandra y M. tejupilcana no existen diferencias significativas (Cuadro 2).

Respecto a los IM, estos variaron entre 276 y 1,380. Las cinco especies presentaron un IM superior a 200, por lo que todas presentan un xilema de tipo mesofítico (Cuadro 2). No obstante, Mimosa tenuiflora es la especie con un mayor IM, aunque no presenta diferencia significativa con $M$. $b a$ hamensis, pero sí con las demás especies; mientras que $M$. leucaenoides es la que tiene el menor IM.

\section{Discusión}

Las cinco especies de Mimosa estudiadas presentaron porosidad difusa. Este tipo de porosidad es común en mimosoideas (Evans et al., 2006) y ha sido considerada por Gilbert (1940, en Moglia y Giménez, 1998) como una ventaja para transportar la escasa cantidad de agua existente en sus hábitats. Además, la presencia de anillos de crecimiento ha sido utilizada para la interpretación del clima (Wimmer y Grabner, 2000) y, generalmente, está asociada a especies que se desarrollan en lugares con estacionalidad marcada (Wheeler et al., 1995). En especies caducifolias, como es el caso de las especies estudiadas, se ha asociado con la generación de hojas nuevas al inicio de la estación de crecimiento, ya que esto demanda la generación de tejidos conductores y, por lo tanto, la formación de anillos de crecimiento (Aguilar-Rodríguez y Barajas-Morales, 2005).

Asimismo, las cinco especies presentan vasos numerosos, lo que concuerda con otras especies de leguminosas como Acacia aroma Gillies ex Hook. \& Arn. (20), A. caven (Mol.) Mol. (23) y A. praecox Griseb. (25); sin embargo, al comparar con Cercidium australe Johnst., el cual presenta en promedio hasta 69 vasos $/ \mathrm{mm}^{2}$, el número de vasos que tienen las especies en estudio no es numeroso, ya que disminuyen hasta en un 50\%. No obstante, todas estas especies tienen en común el habitar en sitios secos (Moglia y Giménez, 1998). De forma adicional, la presencia de diámetros tangenciales pequeños como en Mimosa bahamensis, $M$. hexandra, $M$. leucaenoides y $M$. tejupilcana y medianos en M. tenuiflora, sugiere que su xilema presenta resistencia al estrés hídrico.

De acuerdo con Carlquist (2001), la presencia de vasos de diámetros tangenciales mayores (diámetros medianos 100$200 \mu \mathrm{m}$ y grandes $>200 \mu \mathrm{m}$; IAWA Committee, 1989) y de forma circular, favorece el manejo de grandes cantidades de agua por unidad de tiempo por vaso. Se sugiere que $M i$ mosa tenuiflora se ha especializado para manejar mayores volúmenes de agua, en comparación con las cuatro especies restantes, que presentan vasos más pequeños, indicando una mayor resistencia a la cavitación y, por consiguiente, una menor posibilidad de sufrir embolismos por estrés hídrico (Polanco-Tapia y Grande-Pulido, 2009), y confiriéndole una mayor seguridad a la columna de agua (Woodcock, 1994). En forma adicional, el hecho de que las plantas presenten vasos agrupados ha sido considerado una ventaja, ya que permite una mayor seguridad en la conducción de agua, disminuyendo el riesgo por embolia (Moglia y Giménez, 1998). Los resultados muestran que las cinco especies presentan vasos agrupados; sin embargo, $M$. leucaenoides y $M$. tenuiflora presentaron el mayor número de vasos agrupados, por lo que se propone que ambas especies proveen una mayor seguridad en la conducción de agua.

Erak (1971, en: Metcalfe y Chalk, 1979) reportó que conforme aumenta la altitud sobre el nivel del mar, los vasos presentan paredes más gruesas y sus diámetros son más pequeños, existiendo una correlación negativa con este último carácter. En este estudio, no se reporta una correlación positiva entre la altitud y los diámetros de los vasos, pero sí una correlación negativa entre la altitud y el grosor de la pared (Cuadro 3); es decir, a mayor altitud las paredes de los vasos son más delgadas. Las paredes delgadas de los elementos han sido asociadas a lugares húmedos; sin embargo, las especies estudiadas corresponden a ambientes secos. No obstante, este dato ratifica que el sitio donde se establece Mimosa tejupilcana es el más húmedo (Cuadro 1). Cabe mencionar que los estudios que muestran correlaciones entre la altitud y el grosor de las paredes, en su mayoría, hacen referencia a otros elementos vasculares (p. ej. fibras). 
Cuadro 3. Correlación de Pearson entre cuatro caracteres anatómicos de la madera de las cinco especies de Mimosa (Leguminosae-Mimosoideae) estudiadas y la altitud sobre el nivel del mar. $\varnothing=$ Diámetro

\begin{tabular}{|c|c|c|c|c|c|}
\hline \multirow[t]{2}{*}{ Variables } & \multicolumn{5}{|c|}{$\begin{array}{c}\text { Correlaciones } \\
\text { Correlaciones significativas con } P<0.05\left(^{*}\right)\end{array}$} \\
\hline & $\begin{array}{l}\text { Vasos/ } \\
\text { mm2 }\end{array}$ & $\begin{array}{c}\varnothing \text { tangencial } \\
\text { del vaso } \\
(\mu \mathrm{m})\end{array}$ & $\begin{array}{c}\text { Grosor de } \\
\text { la pared } \\
(\mu \mathrm{m})\end{array}$ & $\begin{array}{l}\text { Longitud de } \\
\text { los elementos } \\
\text { de vaso }(\mu \mathrm{m})\end{array}$ & $\begin{array}{c}\text { Altitud } \\
\text { (m s.n.m.) }\end{array}$ \\
\hline $\begin{array}{l}\varnothing \text { tangencial } \\
\text { del elemento } \\
\text { de vaso }(\mu \mathrm{m})\end{array}$ & $\begin{array}{l}-0.6336 \\
P=0.251\end{array}$ & $\begin{array}{c}1.0 \\
P=----\end{array}$ & $\begin{array}{c}0.8518 \\
P=0.067\end{array}$ & $\begin{array}{c}0.8912^{*} \\
P=0.042\end{array}$ & $\begin{array}{c}-0.6572 \\
P=0.228\end{array}$ \\
\hline Altitud (m) & $\begin{array}{c}0.4208 \\
P=0.480\end{array}$ & $\begin{array}{c}0.6572 \\
P=0.228\end{array}$ & $\begin{array}{c}-0.9463^{*} \\
P=0.015\end{array}$ & $\begin{array}{c}-0.3836 \\
P=0.524\end{array}$ & $\begin{array}{c}1.0 \\
P=----\end{array}$ \\
\hline
\end{tabular}

En Quercus obtusata Humb. \& Bonpl. se reportó una correlación positiva entre la altitud y el grosor de la pared de la fibra; conforme aumenta la altitud, se incrementa el grosor de la pared de la fibra, carácter que ha sido relacionado con la sequía (Chávez-Romero et al., 2010). Asimismo, existen otros estudios que muestran correlaciones negativas entre la altitud y otros caracteres anatómicos. Por ejemplo, Fisher et al. (2007) reportan que en Metrosideros polymorpha Gaudich. al aumentar la altitud, disminuye el diámetro de los vasos, señalando que estos cambios son similares a los provocados por la sequía; este mismo comportamiento lo reporta Yaman (2008) en Juglans regia L.

Las especies estudiadas presentaron vasos numerosos. De acuerdo con León-H. (2001), un mayor número de vasos estaría relacionado con un incremento de la sequía o una disminución de la temperatura del sitio donde éstas se desarrollan. A pesar de tener vasos numerosos, en las cinco especies existe una variación a nivel individuo; no obstante, dichas variaciones no fueron significativas. Las variaciones individuales en el número de vasos $/ \mathrm{mm}^{2}$ han sido reportadas en otras leguminosas como Copaifera langsdorffii Desf. (Marcati et al., 2001), Acacia melanoxylon R.Br. (Wilkins y Papassotiriou, 1989) y Enterolobium contortisiliquum (Vell.) Morong (de Lima et al., 2009). Por otro lado, existe una correlación positiva entre el número de vasos $/ \mathrm{mm}^{2}$ y la altitud; sin embargo, no es significativa, lo que difiere de las observaciones de Baas (1973), quien reportó una correlación negativa entre el número de vasos $/ \mathrm{mm}^{2}$ y la altitud en Ilex. Correlaciones negativas también fueron reportadas por Carlquist (1977) para representantes de las familias Casuarinaceae, Dilleniaceae, Leguminosae, Rutaceae y Violaceae y por Barajas-Morales (1985) para Bombacaceae, Euphorbiaceae, Leguminosae, Myrtaceae y Violaceae.

Longitud de elementos de vaso, placas de perforación y punteaduras intervasculares. Las cinco especies estudiadas presentan elementos de vaso de longitud corta (150 - 221 $\mu \mathrm{m}$, Cuadro 2), lo que corresponde a los ambientes secos en los que se desarrollan; ya que de acuerdo con Novruzova (1972 en Moglia y Gimenez, 1998), la longitud de los elementos de vaso está relacionada con el grado de aridez. En contraste, especies como Chorisia speciosa A.St.-Hill. y Bumelia obtusifolia Roem., representantes de ambientes húmedos que presentan elementos de vaso largos (>350 $\mu \mathrm{m})$.

Además, el hecho de que las especies estudiadas presenten elementos de vaso cortos, les confiere una mayor seguridad en la conducción de agua al disminuir los embolismos; mientras que elementos de vaso largos, otorgan una mayor eficacia conductora (Zimmerman, 1983 en: Carlquist, 2001). Además, los elementos de vaso de longitud corta son considerados los más fuertes a causa de la constricción formada por cada pared del elemento de vaso, aún en aquellos que presentan placa de perforación simple, como es el caso de las cinco especies de Mimosa estudiadas, ya que esto permite resistir grandes presiones y deformaciones relacionadas con las tensiones de la columna de agua (Carlquist, 1975, 2001). La presencia de elementos de vaso cortos es común para las especies que habitan ambientes secos, como es el caso de Acacia subangulata Rose (Abundiz-Bonilla et al., 2004).

Por otro lado, las placas de perforación simple han sido asociadas a especies que se desarrollan en ambientes secos (Moglia y Giménez, 1998) y es un carácter común en la familia Leguminosae (Marchiori, 1996). La presencia de este tipo de placas y la conectividad que se da entre los elementos de vaso genera un aumento en la conductividad hidráulica y, por lo tanto, una mayor vulnerabilidad al embolismo (Polanco-Tapia y Grande-Pulido, 2009). Estos caracteres, asociados a la presencia de punteaduras intervasculares alternas, ofrecen una mayor seguridad a los elementos de vaso, ya que disminuye la conectividad de la red (PolancoTapia y Grande-Pulido, 2009). Se tiene la hipótesis de que las punteaduras intervasculares podrían considerarse una ventaja al disminuir la vulnerabilidad que otorga el tener una placa de perforación simple, ya que éstas permiten el flujo de agua a los vasos adyacentes y, de esta forma, evitan la cavitación (Zweypfenning, 1978). Además, la presencia de punteaduras intervasculares ornamentadas permite que se retengan las burbujas de aire provocadas por una pérdida en la presión de agua (Carlquist, 1982; Choat et al., 2004). De manera adicional, se ha reportado que la estructura de la membrana de la punteadura (grosor y porosidad) juega un papel importante en la resistencia y vulnerabilidad a los embolismos; por ejemplo, el grosor de la membrana de la punteadura influye en el grado de daño de dicha membrana en caso de una deformación mecánica (Jansen et al., 2009), la cual puede atribuirse a las diferencias de presión entre los vasos embolizados y los funcionales (Choat et al., 2004).

Índices de Vulnerabilidad (IV) y Mesomorfía (IM). Los valores de IV y de IM revelan que las cinco especies son poco resistentes al estrés hídrico. Sin embargo, Mimosa leucaenoides presentó el menor IV (1.81), por lo que podría considerarse que presenta madera de tipo xeromórfica; además, 
de las cinco especies, es la más resistente al estrés hídrico: un IV similar lo presenta Geoffroea decorticans (1.5). Mimosa tenuiflora, por presentar un valor de IV superior (6.26), se consideraría la más vulnerable. Mimosa tenuiflora presenta IV similares a los reportados para otras leguminosas como Prosopis ruscifolia Griseb. (5.88), Parkinsonia aculeata L. (5.44) y Cercidium australe (5.00, Moglia y Gimenez, 1998).

Mimosa bahamensis, M. hexandra y M. tejupilcana presentan valores intermedios de IV (Cuadro 2) y son consideradas poco resistentes al estrés hídrico. Estas especies presentan índices de vulnerabilidad similares a Anadenanthera colubrina (Vell.) Bren. (3.03), Caesalpinia paraguariensis Parodi Burk. (3.04), Prosopis nigra Griseb. (3.23) y Gleditsia amorphoides Griseb. (3.64, Moglia y Giménez, 1998).

Los valores de IV estimados en este estudio y los registrados por Carlquist y Hoekman (1985), Lindorf (1994) y Monglia y Gimenez (1998) para otras leguminosas, sugieren que estas especies son vulnerables a la sequía, a pesar de habitar en ambientes secos; lo anterior podría deberse a la presencia de otros órganos vegetales (p. ej. raíces freatófitas, pérdida de hojas) que contrarrestarían la pérdida de agua (Carlquist, 1977). Asimismo, las especies estudiadas presentaron un xilema de tipo mesofítico, con valores de IV menores a siete en todos los casos, lo que señala un sistema de conducción eficaz y seguro para el movimiento del agua (León-H., 2001, 2001-2002).

\section{Conclusiones}

El que la madera de las especies estudiadas presente porosidad difusa, elementos de vaso de diámetro tangencial pequeño, de longitud corta y con placa de perforación simple, contribuye a la seguridad en la conducción de agua. Asimismo, las características de los elementos de vaso de las especies de Mimosa examinadas en este estudio, son semejantes en otras leguminosas, lo que sugiere que la mayoría de las leguminosas poseen seguridad para la conducción de agua.

Las cinco especies de Mimosa presentan características morfológicas de los elementos de vaso que les permiten vivir y adaptarse a los ambientes secos. Esto es relevante ante los escenarios de cambio climático propuestos por el IPCC (Christensen et al., 2007), donde se pronostican cambios en los regímenes de temperatura y precipitación. Se podría esperar que las cinco especies de Mimosa estudiadas resistieran estos cambios. Sin embargo, es preciso tomar en cuenta la etapa de desarrollo, edad y el tiempo de establecimiento de las especies, considerando que las respuestas a cambios de temperatura o precipitación, probablemente, difieren entre niveles de desarrollo. Además, los resultados obtenidos sugieren que las variables consideradas en este trabajo podrían ser relevantes para proponer modelos de distribución espacial de estas cinco especies.

Por otro lado, la seguridad en la conducción de agua que confiere tener vasos numerosos de diámetro pequeño, elementos de vaso de longitud corta y la eficiencia en el uso de agua al presentar elementos de vaso con placas de perforación simple, se ajustan a una madera de tipo xeromórfica. No obstante, las tendencias ecológicas que presentan las cinco especies deben ser analizadas en conjunto con otros aspectos biológicos de las plantas como la fenología. Asimismo, sería importante analizar otros caracteres anatómicos de la madera (p. ej. fibras, traqueidas vasculares); así como examinar la estructura de la hoja y el sistema radical, entre otros, que de manera integral, proporcionarían una mayor información sobre la arquitectura hidráulica de la planta y servirían como indicadores para la selección de especies vegetales con fines de restauración ambiental .

\section{Agradecimientos}

SAMA agradece al Consejo Nacional de Ciencia y Tecnología (CONACyT) por su apoyo (228993/211528) y a Rosaura Grether por la revisión crítica del manuscrito. Además, agradecemos a los revisores anónimos que permitieron mejorar el manuscrito.

\section{Literatura citada}

Abundiz-Bonilla L.A.M., Barajas-Morales J. y Tenorio-Lezama P. 2004. Anatomía de maderas de México: Árboles y arbustos del matorral xerófilo de Tehuacán, Puebla. Universidad Nacional Autónoma de México, México, D.F.

Aguilar-Rodríguez S. y Barajas-Morales J. 2005. Anatomía de la madera de especies arbóreas de un bosque mesófilo de montaña: un enfoque ecológico-evolutivo. Boletín de la Sociedad Botánica de México 77:51-58.

Aguilar-Rodríguez S., Terrazas T. y López-Mata L. 2006. Anatomical wood variation of Buddleja cordata (Buddlejaceae) along its natural range in Mexico. Trees, Structure and Function 20:253-261.

Arias S. y Terrazas T. 2001. Variación en la anatomía de la madera de Pachycereus pecten-aboriginum (Cactaceae). Anales del Instituto de Biología Universidad Nacional Autónoma de México, Serie Botánica 72:157-169.

Baas P. 1973. The wood anatomical range in Ilex (Aquifoliaceae) and its ecological and phylogenetic significance. Blumea 21:193-258.

Baas P., Werker E. y Fahn F. 1983. Some ecological trends in vessel characters. IAWA Bull. n.s. 4:141-158.

Barajas-Morales J. 1985. Wood structural differences between trees of two tropical forests in Mexico. IAWA Bull. n.s. 6:355365.

Barajas-Morales J. y León-Gómez C. 1989. Anatomía de Maderas de México: Especies de una Selva Baja Caducifolia. Universidad Nacional Autónoma de México-Instituto de Biología, México, D.F.

Bernal-Salazar S. y Terrazas T. 2000. Influencia climática sobre la variación radial de caracteres anatómicos de la madera en Abies religiosa. Madera y Bosques 6:73-86.

Camargo-Ricalde S.L. 2000. Descripción, distribución, anatomía, 
composición química y usos de Mimosa tenuiflora (Fabaceae-Mimosoideae) en México. Revista de Biología Tropical 48:939-954.

Camargo-Ricalde S.L., Grether R., Martínez-Bernal A., GarcíaGarcía V. y Barrios del Rosal S. 2001. Especies útiles del género Mimosa (Fabaceae-Mimosoideae) en México. Boletín de la Sociedad Botánica de México 68:33-44.

Carlquist S. 1975. Ecological Strategies of Xylem Evolution. University of California Press, Berkeley.

Carlquist S. 1977. Ecological factors in wood evolution: a floristic approach. American Journal of Botany 64:887-896.

Carlquist S. 1982. Wood anatomy of Onagraceae: further species, root anatomy, significance of vestured pits and allied structures in dicotyledons. Annals of the Missouri Botanical Garden 69:755-769.

Carlquist S. 2001. Comparative Wood Anatomy. Systematic, Ecological and Evolutionary Aspects of Dicotyledon Wood. Springer Verlag. Nueva York.

Carlquist S. y Hoekman D.A. 1985. Ecological wood anatomy of the woody southern Californian Flora. IAWA Bull. n.s. 6:319-347.

Chávez-Romero D.M., Aguilar-Rodríguez S. y Terrazas T. 2010. Variación anatómica en la madera de Quercus obtusata (Fagaceae). Madera y Bosques 16:69-87.

Chehaibar M. y Grether R. 1990. Anatomía de la madera de algunas especies del género Mimosa (Leguminosae). Boletín de la Sociedad Botánica de México 50:3-17.

Choat B., Jansen S., Zwieniecki M.A., Smets E. y Holbrook N.M. 2004. Changes in pit membrane porosity due to deflection and stretching: the role of vestured pits. Journal of Experimental Botany 55:1569-1575.

Christensen J.H., Hewitson B., Busuioc A., Chen A., Gao X., Held I., Jones R., Kolli R.K., Kwon W.-T., Laprise, R. Magaña Rueda V., Mearns L., Menéndez C.G., Räisänen J., Rinke A., Sarr A. y Whetton P. 2007. Regional climate projections. En: Solomon S., Qin D., Manning M., Marquis M., Averyt K.B., Tignor M., LeRoy-Miller H. y Chen Z. Eds. Climate Change: The Physical Science Basis. Summary for Policymarkers. Contribution of Working Group I to the Fourth Assessment Report of the Intergovernmental Panel on Climate Change, pp. 847-940, Cambridge University Press, Cambridge.

De la Barrera E. y Andrade A. 2005. Challenges to plant megadiversity: how environmental physiology can help. New Phytologist 167:5-8.

de Lima R.S., de Oliveira P.L. y Rodrigues L.R. 2009. Anatomia do lenho de Enterolobium contortisiliquum (Vell.) Morong (Leguminosae- Mimosoideae) ocorrente em dois ambientes. Revista Brasileira de Botânica 32:361-374.

Dickison W.C., Rury P.M. y Stebbins G.L. 1978. Xylem anatomy of Hibbertia (Dilleniaceae) in relation to ecology and evolution. Journal of the Arnold Arboretum 59:32-49.

Dhillion S.S., Aguilar-Støen M. y Camargo-Ricalde S.L. 2004. Integrative ecological restoration and local involvement in the Tehuacán-Cuicatlán Valley, Mexico. Environmental Conservation 31:1-3.

Ellerby D.J. y Ennos A.R. 1998. Resistances to fluid flow of model xylem vessels with simple and scalariform perforation plates. Journal of Experimental Botany 49:979-985.

Evans J.A., Gasson P.E. y Lewis G.P. 2006. Wood anatomy of the Mimosoideae (Leguminosae). IAWA J. Suppl. 5:1-117

Fisher B.J., Goldstein G., Jones J.T. y Cordell S. 2007. Wood vessel diameter is related to elevation and genotype in the Hawaiian tree Metrosideros polymorpha (Myrtaceae). American Journal of Botany 94:709-715.

García E. 1973. Modificaciones al Sistema de Clasificación Climática de Köppen (Para Adaptarlo a las Condiciones de la República Mexicana). Universidad Nacional Autónoma de MéxicoInstituto de Geografía, México, D.F.

Giménez A.M. 2004. Anatomía comparada de leño y corteza de Geoffroea striata y Geoffroea decorticans. Madera y Bosques 10:55-68.

Grether R., Camargo-Ricalde S.L. y Martínez-Bernal A. 1996. Especies del género Mimosa (Leguminosae) presentes en México. Boletín de la Sociedad Botánica de México 58:149-152.

Hintze J. 2001. NCSS. Number Cruncher Statistical System, Kaysville.

IAWA Committee. 1989. IAWA list of microscopic features for hardwood identification. IAWA Bull.n. s. 10:219-332.

Jansen S., Baas P., Gasson P. y Smets E. 2003. Vestured pits: do they promote safer water transport? International Journal of Plant Sciences 164:405-413.

Jansen S., Choat B. y Pletsers A. 2009. Morphological variation of intervessel pit membranes and implications to xylem function in angiosperms. American Journal of Botany 96:409-419.

Johansen D.A. 1940. Plant Microtechnique. McGraw Hill, Nueva York.

Lindorf H. 1994. Eco-anatomical wood features of species from a very dry tropical forest. IAWA J. 15:361-376.

León-H. W.J. 2001. Anatomía del leño, aspectos ecológicos y filogenia en mangles de Venezuela. Revista Forestal Venezuelica 45:191-203.

León-H. W.J. 2001-2002. Anatomía xilemática del tronco de Pereskia guamacho (Cactaceae) procedente de Mérida-Venezuela. Pittieria 31:41-49.

León-H. W.J. 2005. Anatomía ecológica del xilema secundario de un bosque seco tropical de Venezuela. Acta Botánica Venezuelica 28:257-274.

Marcati C.R., Angyalossy-Alfonso V. y Benetati L. 2001. Anatomia comparada do lenho de Copaifera langsdorffii Desf. (Leguminosae-Caesalpinoideae) de floresta e cerradão. Revista Brasileira de Botânica 24:311-320.

Marchiori J.N. 1996. Anatomia do xilema secundário de Mimosa incana (Spreng.) Benth. Ciência Florestal 6:53-63.

Martínez-Pérez G., Orozco-Segovia A. y Martorell C. 2006. Efectividad de algunos tratamientos pre-germinativos para ocho especies de la mixteca alta oaxaqueña con características relevantes para la restauración. Boletín de la Sociedad Botánica de México 79:9-20.

Metcalfe C.R. y Chalk I. 1979. Anatomy of the Dicotyledons. Vol. I: Systematic Anatomy of the Leaf and Stem, with a Brief History of the Subject. Clarendon Press, Oxford.

Miller H.J. 1975. Anatomical characteristics of some woody plants of the Angmassalik district of southeast Greenland. Meddelanden Groenlandia 198:1-30.

Moglia G. y Giménez A.M. 1998. Rasgos anatómicos característicos del hidrosistema de las principales especies arbóreas de la Región Chaqueña Argentina. Investigación Agraria 7:53-71.

Montaño-Arias S.A. 2010. Anatomía comparada de la madera de especies arbóreas mexicanas del género Mimosa sección Batocaulon (Leguminosae). Tesis de Maestría, Universidad Autónoma Metropolitana, Unidad Iztapalapa. México, D.F. 141 pp. 
Moya R. y Tomazello.Fo M. 2008. Variation in the wood anatomical structure of Gmelina arborea (Verbenaceae) trees at different ecological conditions in Costa Rica. Revista de Biología Tropical 56:689-704.

Oever van den L., Baas P. y Zande M. 1981. Comparative wood anatomy of Symplocos and latitude and altitude of provenance. IAWA Bull. n.s. 2:3-24.

Pavón N.P., Ballato-Santos J. y Pérez-Pérez C. 2011. Germinación y establecimiento de Mimosa aculeaticarpa var. biuncifera (Fabaceae-Mimosoideae). Revista Mexicana de Biodiversidad 82:653-661.

Polanco-Tapia C. y Grande-Pulido D.C. 2009. Análisis ecoanatómico, evolutivo y comparativo de la madera de 40 especies de dos asociaciones del Bosque altoandino colombiano. Revista Colombia Forestal 12:183-203.

Quintanar-Isaías A., Ángeles G., Zavala-Hurtado J.A. 2009. Anatomía, índices físicos e hidráulicos de la madera de Gliricidia sepium (Jacq.) Steud. Madera y Bosques 15:71-91.

Sidiyasa K. y Baas P. 1998. Ecological and systematic wood anatomy of Alstonia (Apocynaceae). IAWA J. 19:207-229.

Sokal R.R. y Rohlf F.J. 1995. Biometry. WH Freeman, Nueva York.

Sosa V. y Dávila P. 1994. Una evaluación del conocimiento florístico de México. Annals of the Missouri Botanical Garden
81:749-757.

Villagra P.E. y Roig-Juñent F.A. 1997. Wood structure of Prosopis alpataco and $P$. argentina growing under different edaphic conditions. IAWA J. 18:37-51.

Wilkins A.P. y Papassotiriou S. 1989. Wood anatomical variation of Acacia melanoxylon in relation to latitude. IAWA Bull. n.s. 10:201-207.

Wheeler A.E., McClammer J. y LaPasha C.A. 1995. Similarities and differences in dicotyledonous woods of the Cretaceous and Paleocene. San Juan Basin, New Mexico, USA. IAWA J. 16:223-254.

Wimmer R. y Grabner M. 2000. A comparison of tree-ring features in Picea abies as correlated with climate. IAWA J. 21:403-416.

Wodzicki T.J. 2001. Natural factors affecting wood structure. Wood Science and Technology 35:5-26.

Woodcock D.W. 1994. Occurrence of woods with a gradation in vessel diameter across the ring. IAWA J. 15:377-385.

Yaman B. 2008. Variation in quantitative vessel element features of Juglans regia wood in the western black sea region of Turkey. Agrociencia 42:357-365.

Zweypfenning R.C.V.J. 1978. A hypothesis on the function of vestured pits. IAWA Bull. 1:13-15.

Recibido: 13 de enero de 2012

Aceptado: 17 de mayo de 2012 